Objectives: To compare hip fracture risk and major osteoporotic fractures risk using the FRAX® tool, without and with the consideration of asymptomatic VF on VFA. To evaluate the impact of FRAX® calculation and asymptomatic VF identified on VFA on osteoporosis management.

Methods: We conducted a cross-sectional study over a period of 5 months at the rheumatology department. The study included post-menopausal women without a previous diagnosis of VF referred for BMD (Bone mineral density) measurement. Each participant had a BMD assessment and a VFA scan to detect VF. The FRAX® was calculated using femoral neck BMD initially without then with consideration of VF. The change of therapeutic decision was assessed after taking into consideration FRAX $\circledast$ and the VFA results.

Results: The study included 210 post-menopausal women with a mean age of $61.5 \pm 8.5$ years. The mean BMI was $31.04 \pm 5.52 \mathrm{~kg} / \mathrm{m}^{2}$. One women was a current smoker and alcohol intake was not found in our sample. Thirty-seven percent of our participants had at least one fragility fracture. A severe fragility fracture was recorded in $10.5 \%$ and a previous hip fracture was reported in $5.24 \%$. An early menopause was found in $19.5 \%$ of our women. Twenty percent of our population were receiving corticosteroids and $8.2 \%$ of our population had rheumatoid arthritis. The mean vertebral and total hip BMD was $0.955 \pm 0.165 \mathrm{~g} / \mathrm{cm} 2$ and $0.850 \pm 0.135 \mathrm{~g} / \mathrm{cm} 2$ respectively. Osteoporosis and low BMD were found in respectively $50 \%$ and $34.28 \%$. The median probability of major osteoporotic fracture for our population was $1.5 \%$ with an interquartile range from 0.9 to $2.5 \%$ without using VFA data and $1.65 \%$ with an interquartile range from 1 to $2.6 \%$ while taking into consideration VFA results and the difference was statistically significant $(p<0.0001)$. The median probability of hip fracture for our population was $0.4 \%$ with an interquartile range from 0.1 to $0.9 \%$ without using VFA data and $0.4 \%$ with an interquartile range from 0.1 to $1 \%$ while taking into consideration VFA results and the difference was statistically significant $(p<0.0001)$. In all patients, the FRAX® was under the threshold intervention even after including the asymptomatic VF and it did not change the therapeutic decision. The presence of asymptomatic VF on VFA changed the therapeutic decision in $15 \%$ and indicated an anti-osteoporosis drug therapy.

Conclusion: VFA scanning helped in the therapeutic decision in $15 \%$ of our population. In this evaluation, we showed that a comprehensive fracture risk pathway incorporating VFA has enhanced diagnosis of vertebral fractures and improved targeting of treatment better than FRAX® tool.

Disclosure of Interests: None declared.

DOI: 10.1136/annrheumdis-2021-eular.4195

\section{POS1117 AGREEMENT BETWEEN VERTEBRAL FRACTURE ASSESSMENT AND RADIOGRAPHIC SCANS FOR THE DIAGNOSIS OF VERTEBRAL FRACTURE}

C. Daldoul ${ }^{1}$, N. El Amri ${ }^{1}$, K. Baccouche ${ }^{1}$, H. Zegaloui ${ }^{1}$, E. Bouajina ${ }^{1}{ }^{1}$ Farhat Hached Hospital, Rheumatology, Sousse, Tunisia

Background: Conventional radiography of thoracic and lumbar spine are considered the gold-Standard imaging for vertebral fracture (VF) identification. However, there was a growing interest in the use of vertebral fracture assessment (VFA) as a low radiation tool. In fact, the radiation dose for VFA is about 3 micro Sievert compared to 600 Sievert for lateral standard radiography. Moreover, it costs 2 -times less than X-rays. However, the advantage granted by a lower radiation dose is unfortunately counterbalanced by higher noise rates and therefore lower image quality.

Objectives: The aim of this study was to investigate the diagnostic accuracy of VFA compared to lateral spine radiographs.

Methods: We conducted a cross-sectional study over a period of 5 months at the rheumatology department. The study included post-menopausal women without a previous diagnosis of VF. Each participant had a BMD assessment, a VFA scan and a lateral thoracolumbar X-rays $(R x)$ to detect VF. VF were identified according the Genant semi-quantitative method. The number of unreadable vertebrae were compared between VFA and Rx using a McNemar test. Cohen's kappa coefficient, the sensitivity (Se), the specificity (Sp), the positive (PPV) and negative predictive (NPV)values at each vertebral level and at the individual level were calculated with $95 \%$ confidence interval $(95 \% \mathrm{Cl})$ to analyze the agreement between VFA and $R x$ results.

Results: The study included 62 patients were collected. The mean age was $62.03 \pm 7.84$ years. the mean body mass index (BMI) was $29.99 \pm 5.13 \mathrm{~kg} / \mathrm{m}^{2}$ and the mean menopausal duration was $15.7 \pm 8.28$ years. Parental history of hip fracture and prior history of fragility fracture were recorded in $25.9 \%$ and $40.3 \%$ respectively. A premature menopause, Rheumatoid arthritis and use of corticosteroids were found in $12.9 \%, 4.8 \%$ and $19.4 \%$ respectively. A historical height loss of more than $4 \mathrm{~cm}$ and a prospective height loss of more than $2 \mathrm{~cm}$ were reported in $21 \%$ and $38.7 \%$ respectively. Using VFA, $22.6 \%$ of our population had at least one $V F \geq$ grade 2 and $33.9 \%$ had at least one $V F \geq$ grade 1 . Using $R x$, $19.4 \%$ of our population had at least one $V F \geq$ grade 2 and $25.8 \%$ had at least one $V F \geq g r a d e 1$. Among all vertebral levels, no statistically significant difference was found while comparing the number of unreadable vertebrae on VFA and radiographs. Taking into consideration $\mathrm{VF} \geq$ grade2, there was an almost perfect agreement between VFA and $\mathrm{Rx}(\mathrm{k}=0.806, \mathrm{p}<0.001)$; the Se, Sp, PPV and NPV of VFA were respectively $91.7 \%$ (95\% Cl 59.8-99.6), 94\%(95\% Cl 82.5-98.4), $78.6 \%(95 \% \mathrm{Cl} 48.8-94.3)$ and $97.9 \%(95 \% \mathrm{Cl} 87.5-99.9)$. . Taking into consideration $V F \geq g$ rade1, there was a moderate agreement between VFA and $R x$ $(\mathrm{k}=0580, \mathrm{p}<0.001)$; the Se, Sp,PPV and NPV of VFA were respectively $81.3 \%$ (95\% Cl 53.7-95),82.6\% (95\% Cl 68-91.7), 61.9\% (95\% Cl 38.7-81) and $92.7 \%$ $(95 \% \mathrm{Cl} 79-98.1)$. At the vertebral level and when including only grade 2 and 3 VF, the Se, Sp, PPV of VFA were $100 \%$ (95\% Cl 82.2-100), 98.9\% (95\% C 97.7-99.5), $74.2 \%(95 \% \mathrm{Cl} 55.1-87.5)$ and $100 \%$ (95\% Cl 99.3-100) respectively. At the vertebral level, the Se, Sp, PPV and NPV of VFA in detection of grade $\geq 1$ VF were 65.8\% (95\% Cl 48.6-79.9), 98.3\% (95\% Cl 96.9-99.1), 67.6\% (95\% C $50.1-81.4)$ and $98.1 \%(95 \% \mathrm{Cl} 96.8-99.0)$ respectively. The analysis by vertebral level revealed substantial to almost perfect agreement for all levels except for T4 and T6 where the test was not applicable. There was a perfect agreement for T5 T8, L3 and L4 vertebrae.

Conclusion: VFA showed a high diagnostic performance compared to Rx. One interesting finding is that the NPV of VFA is very high at the vertebral and the individual level and either grade 1 VF were took into consideration or not. This means that a normal VFA can formally rule out the presence of VF.

Disclosure of Interests: None declared.

DOI: 10.1136/annrheumdis-2021-eular.4206

\section{POS1118 AND TREATMENT FOR DOCTORS WORKING IN THE FIELD OF PHYSICAL AND REHABILITATION MEDICINE}

L. Marchenkova ${ }^{1}$, V. Vasileva ${ }^{1} .{ }^{1}$ National Medical Research Center for Rehabilitation and Balneology of Ministry of Health of Russian Federation, Rehabilitation Department for Somatic Patients, Moscow, Russian Federation

Background: There is a high prevalence of osteoporosis (OP) among patients of the older age undergoing rehabilitation. Therefore, it is obvious that physicians working in the field of physical and rehabilitative medicine should be well oriented in this medical problem.

Objectives: to study the relevance of the problem of osteoporosis (OP) for physicians working in the field of physical and rehabilitation medicine, their awareness of the main methods of diagnosis, treatment and prevention of this disease, as well as the frequency of their use in daily clinical activities.

Methods: A cross-type study was carried out using a questionnaire survey. The study included 157 doctors (M-34, F-123) of 8 medical specialties working in 27 specialized medical institutions on the profile of "medical rehabilitation. The questionnaire for doctors consisted of 21 items of special questions.

Results: $90.45 \%$ of the surveyed doctors believed that the problem of OP is relevant for their clinical activities, $100 \%$ of the respondents indicated that the presence of OP significantly affects the rehabilitation prognosis and $95.54 \%$ - on the degree of effectiveness of medical rehabilitation. According to the respondents, patients with OP make up on average $30.0 \%[20.0 ; 50.0](0-90)$ of the total flow of patients. $92.36 \%(145 / 157)$ of doctors indicated that they know the risk factors for OP, 98.73\% (155/157) - methods for diagnosing OP, 68.79\% (108/157) - methods for treating OP, $80.25 \%(126 / 157)$ - methods of preventing OP, $47.13 \%$ (74/157) - what is FRAX. However, $35.01 \%$ (55/157) of the respondents considered their level of awareness of the problem sufficient for managing patients with OP. Diagnostic procedures for OP are recommended by all endocrinologists $(100 \%)$ and the majority of traumatologists $(72.73 \%)$, gynecologists (66.67) and cardiologists $(64.28 \%)$, as well as on average half $(50 \%)$ neurologists and therapists. Endocrinologists (100\%), gynecologists (66.67\%) and therapists (60\%) are mainly involved in the treatment of OP. $32.48 \%$ (51/157) of physicians have ever referred their patients to a bone mineral density assessment.

Conclusion: Conclusion. The problem of OP is relevant for the clinical activities of specialists in physical and rehabilitation medicine, and there is the need for advanced training on the problem of OP among these specialists.

Disclosure of Interests: None declared.

DOI: 10.1136/annrheumdis-2021-eular.4274

\section{\begin{tabular}{|l|l}
\hline POS1119 & A SINGLE DOSE OF ZOLEDRONATE INDUCES \\
\hline
\end{tabular} MODIFICATIONS OF SERUM VEGF IN OSTEOPOROTIC POSTMENOPAUSAL WOMEN}

F. Bellone $^{1}$, N. Morabito ${ }^{1}$, A. Gaudio ${ }^{2}$, A. Sottile ${ }^{1}$, S. Loddo ${ }^{1}$, F. Corica ${ }^{1}$, A. Catalano ${ }^{1}{ }^{1}$ University of Messina, Department of Clinical and Experimental Medicine, Messina, Italy; ${ }^{2}$ University of Catania, Department of Clinical and Experimental Medicine, Catania, Italy

Background: Zoledronate ( $\mathrm{Zol}$ ) is an aminobisphosphonate commonly used to treat osteoporosis and other benign and malignant skeletal diseases. Exposure to bisphosphonates has been previously associated with the risk of osteonecrosis of the jaw (BRONJ), a rare but serious side effect. In cancer patients 